\title{
The least core, kernel and bargaining sets of large games ${ }^{\star}$
}

\author{
Ezra Einy ${ }^{1,2}$, Dov Monderer ${ }^{3}$, and Diego Moreno ${ }^{1}$ \\ ${ }^{1}$ Departamento de Economía, Universidad Carlos III de Madrid, \\ E-28903 Getafe, Madrid, SPAIN \\ ${ }^{2}$ Department of Economics, Ben-Gurion University of the Negev, Beer Sheva, ISRAEL \\ ${ }^{3}$ Faculty of Industrial Engineering and Management, Technion-Israel Institute of Technology, \\ Haifa, ISRAEL
}

Received: June 6, 1996; revised version: March 1, 1997

Summary. We study the least core, the kernel and bargaining sets of coalitional games with a countable set of players. We show that the least core of a continuous superadditive game with a countable set of players is a nonempty (norm-compact) subset of the space of all countably additive measures. Then we show that in such games the intersection of the prekernel and the least core is non-empty. Finally, we show that the Aumann-Maschler and the Mas-Colell bargaining sets contain the set of all countably additive payoff measures in the prekernel.

\section{JEL Classification Number: C71.}

\section{Introduction}

Solution concepts for coalitional games with an infinite set of players have been studied in many works. Most of these works deal with the core and the Shapley value. Bargaining sets and related solution concepts for games with a finite set of players have been studied intensively (for a comprehensive survey see Maschler [11]). There are a few works concerning bargaining sets (and related concepts) of games with an infinite set of players. Wesley [21] deals with the kernel of games with a countable set of players. Bird [3] studies the nucleolus-like solutions for games with a measurable space of players. Mas-Colell [14] introduces his bargaining set in the context of pure exchange

\footnotetext{
^ The first author thanks the Spanish Ministry of Education for its support. The third author gratefully acknowledges financial support from the Ministerio de Trabajo y Asuntos Sociales through funds administered by the Cátedra Gumersindo Azcárate, and from DGICYT grant PB93-2030. This work was done while Einy and Monderer visited the Department of Economics of the Universidad Carlos III de Madrid. The support of the Department is gratefully acknowledged.
} 
economies with a continuum of agents. Shitovitz [19] deals with the MasColell bargaining set in mixed market games. Einy et al. [8] study the Mas-Colell bargaining set in convex games with a measurable space of players.

The present work deals with the least core, the kernel, and the AumannMaschler and Mas-Colell bargaining sets for superadditive games with a countable set of players. The least core was introduced by Maschler, Peleg and Shapley [13], where they study its relation to the kernel and the nucleolus. It is well known that the core of a continuous game with a measurable space of players, if it is non-empty, consists of countably additive payoff measures (e.g., Schmeidler [17]). Here we show that the least core of a continuous superadditive game with a countable set of players is a nonempty norm-compact subset of the set of all countably additive measures defined on the set of coalitions (see Theorem A).

The kernel of a cooperative game was introduced by Davis and Maschler [5]. Since then it has been the subject of many studies. Originally it was regarded as an auxiliary solution concept whose main task was to illuminate the properties of the Aumann-Maschler bargaining set. Nevertheless, the kernel possesses interesting mathematical properties, and reflects in many ways the structure of the game. The prekernel is a simplified version of the kernel which is not restricted to individually rational payoffs. We show that in continuous superadditive games with a countable set of players the prekernel (and hence the kernel) and the least core have a non-empty intersection. The proof of this result uses finite approximations. Wesley [21] proved by using non-standard analysis that under some conditions the kernel of a superadditive game with a countable set of players is non-empty (for every coalition structure). We show that Wesley's conditions imply that the game is continuous. We also give an example of a continuous game which does not satisfy one of Wesley's conditions (see Lemma 4.3 and Example 4.4). Thus, Wesley's result is a special case of our result when the coalition structure includes only the grand coalition.

The first definition of a bargaining set for cooperative games was given in Aumann and Maschler [1]. Recently, several new concepts of bargaining set have been introduced (see Maschler [11] for a survey). Davis and Maschler [4] and Peleg [15] proved that the Aumann-Maschler bargaining set is nonempty in a coalitional game with a finite set of players. We show that in continuous superadditive games with a countable set of players, the Aumann-Maschler bargaining set contains the set of all countably additive payoff measures in the prekernel, and thus it always contains a countably additive payoff measure (see Theorem C).

Mas-Colell [14] proposed a bargaining set which is a modification of the Aumann-Maschler bargaining set. One of the advantage of the Mas-Colell bargaining set is that it can be defined for games with a continuum of players. Mas-Colell [14] showed that in atomless pure exchange economies his bargaining set coincides with the set of competitive equilibria, and he pointed out that in finite coalitional games, the prekernel is always contained in his 
bargaining set (see also Vohra [20]). In the definition of the Mas-Colell bargaining set it is not assumed that payoffs are individually rational. We show that in continuous superadditive games with a countable set of players the Mas-Colell bargaining set contains the set of all countably additive payoff measures in the prekernel, and thus it always contains an individually rational countably additive payoff measure.

The paper is organized as follows. Section 2 contains the basic definitions and the preliminary results which are relevant to our work. In Section 3 we prove that the least core of a continuous superadditive game with a countable set of players is a non-empty norm-compact subset of the space of all countably additive measures on the set of coalitions. In Section 4 we show that in continuous superadditive games with a countable set of players the least core and the prekernel have a non-empty intersection. In Section 5 we prove that in continuous superadditive games the bargaining set of Aumann and Maschler and that of Mas-Colell contain the set of all countably additive payoff measures in the prekernel.

\section{Basic definitions and preliminary results}

In this section we define some basic notions we use throughout and prove some preliminary results.

\subsection{Mathematical preliminaries}

Let $N$ be the set of natural numbers. The set of subsets of $N$ is denoted by $2^{N}$. The set of all functions $f: N \rightarrow\{0,1\}$ is denoted by $\{0,1\}^{N}$. Note that $\{0,1\}^{N}$ is a compact metric space (as the product of countable metric spaces), and convergence of sequences in this metric is identical to pointwise convergence. Every $S \in 2^{N}$ can be naturally identified with its indicator function $1_{S} \in\{0,1\}^{N}$. The correspondence $S \longleftrightarrow 1_{S}$ induces a metric on $2^{N}$ under which it is compact. It is well known that a sequence $\left\{S_{n}\right\}_{n}^{\infty}$ converges to $S$ (written $\lim _{n \rightarrow \infty} S_{n}=S$ ) in this metric if and only if

$$
S=\underline{\lim }_{n \rightarrow \infty} S_{n}=\varlimsup_{n \rightarrow \infty} S_{n},
$$

where $\underline{\lim }_{n \rightarrow \infty} S_{n}=\bigcup_{n 1}^{\infty} \bigcap_{k n}^{\infty} S_{k}$, and $\varlimsup_{n \rightarrow \infty} S_{n}=\bigcap_{n 1}^{\infty} \bigcup_{k n}^{\infty} S_{k}$.

If $\left\{S_{n}\right\}_{n 1}^{\infty}$ is a sequence of sets in $2^{N}$ such that $S_{n} \subset S_{n+1}$, and $\bigcup_{n}^{\infty} S_{n}=S$ we write $S_{n} \nearrow S$. Similarly, if $\left\{S_{n}\right\}_{n}^{\infty}$ is a sequence of sets in $2^{N}$ such that $S_{n+1} \subset S_{n}$, and $\bigcap_{n 1}^{\infty} S_{n}=S$, then we write $S_{n} \searrow S$. Note that if $\left\{S_{n}\right\}_{n 1}^{\infty}$ is a sequence such that $S_{n} \nearrow S$ or $S_{n} \searrow S$, then $\lim _{n \rightarrow \infty} S_{n}=S$. A function $v: 2^{N} \rightarrow \Re$ is monotonic if $v(S) \leq v(T)$ whenever $S \subset T$. A function $v$ on $2^{N}$ is continuous if it is continuous with respect to the natural topology on $2^{N}$ defined above. For monotonic functions we have the following characterization of continuity.

Lemma 2.1. Let $v: 2^{N} \rightarrow \Re$ be a monotonic function. Then $v$ is continuous if and only if for every $S \in 2^{N}$ we have $\lim _{n \rightarrow \infty} v\left(S_{n}\right)=v(S)$ whenever $S_{n} \nearrow S$ or $S_{n} \searrow S$. 
Proof. We prove the non-obvious part of the lemma. Let $S \in 2^{N}$. Assume $\lim _{n \rightarrow \infty} v\left(S_{n}\right)=v(S)$ whenever $S_{n} \nearrow S$ or $S_{n} \searrow S$. We show that $v$ is continuous at $S$. Let $\left\{S_{n}\right\}_{n}^{\infty}$ be such that $\lim _{n \rightarrow \infty} S_{n}=S$; that is $S=\underline{\lim }_{n \rightarrow \infty} S_{n}=$ $\varlimsup_{n \rightarrow \infty} S_{n}$. We show that $\lim _{n \rightarrow \infty} v\left(S_{n}\right)=v(S)$. For each $n$ let $A_{n}=\bigcap_{k}^{\infty} S_{k}$, and $B_{n}=\bigcup_{k}^{\infty} S_{k}$. As $v$ is monotonic and $A_{n} \subset S_{n} \subset B_{n}$ for each $n$, then $v\left(A_{n}\right)$ $\leq v\left(S_{n}\right) \leq v\left(B_{n}\right)$ for each $n$. Now, $A_{n+1} \supset A_{n}$ and $S=\underline{\lim } S_{n}=\bigcup_{n}^{\infty} A_{n}$. Also $B_{n+1} \subset B_{n}$, and $S=\varlimsup \lim S_{n}=\bigcap_{n 1}^{\infty} B_{n}$. Thus, $A_{n} \nearrow S$ and $B_{n} \searrow S$. Hence by our assumption

$$
\lim _{n \rightarrow \infty} v\left(A_{n}\right)=\lim _{n \rightarrow \infty} v\left(B_{n}\right)=v(S),
$$

and therefore $\lim _{n \rightarrow \infty} v\left(S_{n}\right)=v(S)$.

We now recall some standard definitions and results from measure theory which are used throughout the paper. A finitely additive measure on $2^{N}$ is a function $\mu: 2^{N} \rightarrow \Re$ which satisfies $\mu(A \cup B)=\mu(A)+\mu(B)$ whenever $A$ and $B$ are two disjoint subsets of $N$. The measure $\mu$ is called countably additive if for every countable family $\left\{A_{i}\right\}_{i}^{\infty}$ of disjoint subsets of $N$ we have $\mu\left(\bigcup_{i 1}^{\infty} A_{i}\right)=\sum_{i 1}^{\infty} \mu\left(A_{i}\right)$. It is well known that $\mu$ is countably additive iff it is continuous at $N$. A finitely additive measure $\mu$ on $2^{N}$ is called purely finitely additive if there is no non-negative and non-zero countably additive measure $\lambda$ such that $\lambda(A) \leq \mu(A)$ for all $A \in 2^{N}$. We note that if $\mu$ is non-negative, then it is purely finitely additive iff it vanishes at every finite subset of $N$. A theorem of Yosida and Hewitt (e.g., Theorem 1.23 of Yosida and Hewitt [22]) asserts that any finitely additive measure can be uniquely decomposed into a sum of a countably additive measure and a purely finitely additive measure.

The Banach space of all bounded finitely additive measures on $2^{N}$ with the variation norm is denoted by $b a$. Its closed subspace consisting of all countable additive measures is denoted by $c a$. It is well known that $b a$ is naturally identified with the dual space of $l_{\infty}=l_{\infty}(N)$, which is the Banach space of all bounded sequences of real numbers. Also, $\mathrm{ca}$ can be naturally identified with $l_{1}=l_{1}(N)$; i.e., every $x \in l_{1}$ corresponds to $\mu_{x} \in c a$, where

$$
\mu_{x}(S)=\sum_{i \in S} x_{i}
$$

for every $S \in 2^{N}$.

The following lemma plays an important role in our work.

Lemma 2.2. Let $K$ be a weak*-compact subset of ba that contains only countably additive measures (i.e., $K \subset c a$ ). Then $K$ is norm-compact.

Proof. Since $K \subset c a$, we can view $K$ as a subset of $\ell_{1}$. As $b a$ is the norm-dual of $\ell_{\infty}$, which in turns is the norm-dual of $\ell_{1}$, the weak* topology induced by $b a$ on $K$ coincides with the weak topology induced by $\ell_{1}$ on $K$. Hence $K$ is a weakly compact subset of $\ell_{1}$. Since $\ell_{1}$ is a separable Banach space, the weak topology on its weakly compact subsets is metrizable (see e.g., Theorem 3.V.6.3 in Dunford-Schwartz [7]). Therefore $K$ is weakly sequentially 
compact in $\ell_{1}$. By Schur's Theorem (see Diestel [6], page 85, and Corollary 14 page 296 of Dunford-Schwartz [7]), weak convergence and norm convergence of sequences are equivalent in $\ell_{1}$; hence $K$ is norm-compact in $\ell_{1}$.

We conclude this subsection with some definitions concerning nets which will be used in the proof of Theorem B.

A directed system is a pair $(D, \preceq)$, where $D$ is a set and $\preceq$ is a binary relation on $D$ such that the following holds:

(a) If $\alpha \preceq \beta$ and $\beta \preceq \gamma$, then $\alpha \preceq \gamma$.

(b) If $\alpha, \beta \in D$, then there exists $\gamma \in D$ such that $\alpha \preceq \gamma$ and $\beta \preceq \gamma$.

A net is a mapping of a directed system into a topological space $X$. We usually write $x_{\alpha}$ for the value of the net at $\alpha \in D$ and $\left\{x_{\alpha} \mid \alpha \in D\right\}$ (or simply $\left.\left\{x_{\alpha}\right\}\right)$ for the net itself. We say that $\left\{x_{\alpha}\right\}$ converges to a point $x \in X$ if for every open set $O$ containing $x$ there exists $\alpha_{0} \in D$ such that $x_{\alpha} \in O$ for all $\alpha \succeq \alpha_{0}$. Let $\left\{x_{\alpha} \mid \alpha \in D\right\}$ be a net. For every $\alpha \in D$ let $\beta_{\alpha} \in D$ be such that $\beta_{\alpha} \succeq \alpha$; then the set $\left\{x_{\beta_{\alpha}} \mid \alpha \in D\right\}$ is called a subnet of $\left\{x_{\alpha} \mid \alpha \in D\right\}$.

For a further discussion of nets and related notions the reader is referred to Kelley [10], p. 6572.

\subsection{Game theoretic preliminaries}

We refer to the members of $N$ as players, and to each subset of $N$ as a coalition. A game is a bounded function $v: 2^{N} \rightarrow \Re$ satisfying $v(\emptyset)=0$, and for which there is $\lambda \in c a$ such that $v(S) \geq \lambda(S)$ for every $S \in 2^{N}$. (Note that the later requirement is satisfied for every non-negative set function $v$ with $\lambda \equiv 0$.) A game $v$ is superadditive if $v(S \cup T) \geq v(S)+v(T)$ for every two disjoint coalitions $S, T \in 2^{N}$. Throughout this paper we assume that all games under consideration are superadditive, but to avoid confusion we emphasize it in the statements of the results. Note that every $\lambda \in c a$ is continuous, and that if $v(S) \geq \lambda(S)$ for all $S$, then $v-\lambda$ is a monotonic function. Hence by Lemma 2.1 a game $v$ is continuous on $2^{N}$ if and only if $\lim _{n \rightarrow \infty} v\left(S_{n}\right)=v(S)$ whenever $S_{n} \nearrow S$ or $S_{n} \searrow S$. Note that our definition of continuity coincides with that of Schmeidler [17] (see also Aumann and Shapley [2]).

For every game $v$ and every coalition $S \in 2^{N}$, we define

$$
\sigma_{v}(S)=\inf \sum_{i}^{n} v\left(S_{i}\right)
$$

where infimum is taken over all finite partitions $\left\{S_{1}, S_{2}, \ldots, S_{n}\right\}$ of $S$. Note that $\sigma_{v}(i)=v(\{i\})$ for every $i \in N$. Note also that $\sigma_{v} \in b a$. Moreover, $\sigma_{v}$ is the largest measure in $b a$ such that $\sigma_{v}(S) \leq v(S)$ for all $S \in 2^{N}$; that is, if $\lambda \in b a$ and $\lambda(S) \leq v(S)$ for all $S \in 2^{N}$, then $\lambda \leq \sigma_{v}$ (pointwise). Also, if $v$ is continuous then $\sigma_{v} \in c a$.

A payoff measure (or a preimputation) is a measure $\lambda \in b a$ such that $\lambda(N)=v(N)$. A payoff measure $\lambda$ is individually rational if $\lambda \geq \sigma_{v}$. An imputation is an individually rational payoff measure. The set of all imputations is denoted by $I(v)$. 


\section{The least core}

Recall that the core of a game $v$, denoted by $C(v)$, is the set off all imputations $\mu \in I(v)$ such that $\mu(S) \geq v(S)$ for each $S \in 2^{N}$. For every $\lambda \in b a$ let $f(\lambda)=\sup \{v(S)-\lambda(S) \mid S \subset N\}$. The least core of the game $v$, denoted $L C(v)$, is the set of all imputations $\mu \in I(v)$ for which $f$ attains its minimal value on $I(v)$. Note that since $f$ is the supremum of affine weak ${ }^{*}$-continuous functions, it is a convex and lower semicontinuous function on $b a$. As $I(v)$ is a weak*-compact subset of $b a, f$ attains its minimal value $\epsilon_{v}$ on $I(v)$. Therefore $L C(v)$ is a non-empty convex weak*-compact subset of $I(v)$. We note that $\epsilon_{v} \geq 0$, and $\epsilon_{v}=0$ if and only if $C(v) \neq \emptyset$. In this case $C(v)=L C(v)$.

Theorem A. Let $v$ be a superadditive continuous game. Then $L C(v)$ is a nonempty convex norm-compact subset of ca.

Proof. By Lemma 2.2 it suffices to prove that $L C(v) \subset c a$. Without loss of generality (w.l.o.g.) assume that $\sigma_{v}$ is identically zero. (Alternatively, we could replace the game $v$ with the game $w=v-\sigma_{v}$, which is also continuous and satisfies $\mu \in L C(w)$ if and only if $\mu+\sigma_{v} \in L C(v)$.) Also w.l.o.g. assume that $v(N)=1$. Finally, we may assume that $\epsilon_{v}>0$, as otherwise the result follows from Schmeidler [17].

Let $\mu \in L C(v)$. By Theorem 1.23 of Yosida and Hewitt [22], $\mu$ can be uniquely decomposed into a sum of a non-negative countably additive measure $\mu_{c}$ and a non-negative purely finitely additive measure $\mu_{p}$ (i.e., a measure $\mu_{p}$ satisfying $\mu_{p}(S)=0$, for each finite $S \in \Sigma$ ). We must show that $\mu_{p}$ is identically zero. As $\mu_{p}$ is non-negative, it suffices to show that $\mu_{p}(N)=0$. Assume that $\mu_{p}(N)>0$. We show that this implies that $\max \left\{v(S)-\mu_{c}(S)\right.$ $\left.\mid S \in 2^{N}\right\}>\epsilon_{v}$. This leads to a contradiction since $\max \left\{v(S)-\mu_{c}(S)\right.$ $\left.\mid S \in 2^{N}\right\}$ (which exists because $v-\mu_{c}$ is continuous) is bounded above by $\epsilon_{v}$. Indeed, let $S \in 2^{N}$, and for every $n \in N$ define $S_{n}=\{1, \ldots, n\}$. Then

$$
\begin{aligned}
v(S)-\mu_{c}(S) & =\lim _{n \rightarrow \infty}\left(v\left(S \cap S_{n}\right)-\mu_{c}\left(S \cap S_{n}\right)\right) \\
& =\lim _{n \rightarrow \infty}\left(v\left(S \cap S_{n}\right)-\mu\left(S \cap S_{n}\right)\right) \\
& \leq \epsilon_{v} .
\end{aligned}
$$

In the remaining of the proof of Theorem A assume that $\mu_{p}(N)>0$. The proof proceeds in several steps.

STEP 1: We show that $\mu_{c}(N)>0$.

Assume by way of contradiction that $\mu_{c}(N)=0$. Then $\mu=\mu_{p}$. As $\mu \in L C(v), v(S)-\mu(S) \leq \epsilon_{v}$, for every $S \in 2^{N}$. As $v$ is continuous, we have $\lim _{n \rightarrow \infty} v\left(S_{n}\right)=v(N)=1$. Since $\mu=\mu_{p}$ and $\mu_{p}\left(S_{n}\right)=0$, we have $v\left(S_{n}\right)-\mu\left(S_{n}\right)$ $=v\left(S_{n}\right)$, for each $n$. Therefore $\epsilon_{v} \geq v\left(S_{n}\right)$ for each $n$, and thus $\epsilon_{v}=1$. Let $\xi \in I(v)$ be given for each $S \in 2^{N}$ by $\xi(S)=\sum_{i \in S} 2^{-i}$. As $v-\xi$ is continuous, there is $S^{*} \in 2^{N}$ such that $v\left(S^{*}\right)-\xi\left(S^{*}\right)=\max \left\{v(S)-\xi(S) \mid S \in 2^{N}\right\}$. From the definition of $\epsilon_{v}$ it is clear that $1=\epsilon_{v} \leq v\left(S^{*}\right)-\xi\left(S^{*}\right)$; hence $S^{*} \neq \emptyset$. Also 
$v(S) \leq 1$, and $\xi(S)>0$ for each $S \neq \emptyset$ in $2^{N}$. Summing up these inequalities we have

$$
1=\epsilon_{v} \leq v\left(S^{*}\right)-\xi\left(S^{*}\right) \leq 1-\xi\left(S^{*}\right)<1,
$$

which is a contradiction. Hence $\mu_{c}(N)>0$.

STEP 2: We show that the payoff measure $\lambda=\frac{1}{\mu_{c}(N)} \mu_{c}$ is a member of $L C(v)$.

Let $\alpha=\frac{1}{\mu_{c}(N)}$. As $\mu_{p}(N)>0, \alpha>1$. For each $S \in 2^{N}$ we have

$$
v(S)-\lambda(S)=v(S)-\alpha \mu_{c}(S)=v(S)-\mu_{c}(S)-(\alpha-1) \mu_{c}(S) \leq v(S)-\mu_{c}(S) .
$$

Recall that $S_{n}=\{1, \ldots, n\}$. Then for all $S \in 2^{N}$ we have

$$
\begin{aligned}
v(S)-\lambda(S) & =\lim _{n \rightarrow \infty}\left(v\left(S \cap S_{n}\right)-\lambda\left(S \cap S_{n}\right)\right) \\
& \leq \lim _{n \rightarrow \infty}\left(v\left(S \cap S_{n}\right)-\mu_{c}\left(S \cap S_{n}\right)\right) \\
& =\lim _{n \rightarrow \infty}\left(v\left(S \cap S_{n}\right)-\mu\left(S \cap S_{n}\right)\right) \\
& \leq \epsilon_{v} .
\end{aligned}
$$

Therefore $\lambda \in L C(v)$.

STEP 3: We show that $\max \left\{v(S)-\mu_{c}(S) \mid S \in 2^{N}\right\}>\epsilon_{v}$, and we will get the desired contradiction.

For every $S \in 2^{N}$ we have $v(S)-\mu_{c}(S) \geq v(S)-\mu(S)$. As $\mu \in L C(v)$, we have $\sup \left\{v(S)-\mu(S) \mid S \in 2^{N}\right\}=\epsilon_{v}$. Hence $\max \left\{v(S)-\mu_{c}(S) \mid S \in 2^{N}\right\} \geq \epsilon_{v}$. We show that this inequality is strict. Assume to the contrary that $\max \left\{v(S)-\mu_{c}(S) \mid S \in 2^{N}\right\}=\epsilon_{v}$. Let $\lambda=\frac{1}{\mu_{c}(N)} \mu_{c}$; by Step 2, $\lambda \in L C(v)$. Now if $S \in 2^{N}$ is such that $v(S)-\lambda(S)=\epsilon_{v}$, then $\lambda(S)=0$. For otherwise we would have $v(S)-\mu_{c}(S)>v(S)-\lambda(S)=\epsilon_{v}$. Let $S_{0}=\{i \in N \mid \lambda(\{i\})=0\}$. Because $\lambda \in c a, \lambda\left(S_{0}\right)=0$. Let $\bar{S} \in 2^{N}$ be such that $v(\bar{S})-\lambda(\bar{S})=\epsilon_{v}(\bar{S} \neq \emptyset$ because $\epsilon_{v}>0$ ); hence $\lambda(\bar{S})=0$, and therefore $\bar{S} \subset S_{0}$. Thus, $S_{0} \neq \emptyset$.

Let $j \in N \backslash S_{0}$. Then $\lambda(\{j\})>0$. Let $\mathscr{C}_{j}=\left\{S \in 2^{N} \mid j \in S\right\}$. The set $\left\{1_{S} \mid S \in \mathscr{C}_{j}\right\}$ is a closed subset of $\{0,1\}^{N}$ in the topology of pointwise convergence, and therefore it is compact. Let $Q \in \mathscr{C}_{j}$ be such that $v(Q)$ $-\lambda(Q)=\max \left\{v(S)-\lambda(S) \mid S \in \mathscr{C}_{j}\right\}$. Let $\delta=v(Q)-\lambda(Q)$. If $\delta=\epsilon_{v}$, then $\lambda(Q)=0$, which is impossible since $j \in Q$, and therefore $\lambda(Q) \geq \lambda(\{j\})>0$. Hence $\delta<\epsilon_{v}$. Let $0<\epsilon<\min \left(\lambda(\{j\}), \epsilon_{v}-\delta\right)$, and for each $i \in S_{0}$ let $\epsilon_{i}=\frac{\epsilon 2^{i}}{\sum_{l \in S_{0}}{ }^{2}{ }^{\prime}}$. Also for each $i \in N$ define

$$
\hat{\lambda}(\{i\})=\left\{\begin{array}{ll}
\epsilon_{i} & i \in S_{0} \\
\lambda(\{j\})-\epsilon & i=j \\
\lambda(\{i\}) & i \notin S_{0} \cup\{j\}
\end{array} .\right.
$$

Note that $\hat{\lambda} \in c a_{+}$. Moreover, since $\hat{\lambda}\left(S_{0}\right)=\epsilon$, we have $\hat{\lambda}(N)=1$, and therefore $\hat{\lambda} \in I(v)$. We show that $\max \left\{v(S)-\hat{\lambda}(S) \mid S \in 2^{N}\right\}<\epsilon_{v}$, and this 
will contradict the definition of $\epsilon_{v}$. Let $S \in 2^{N}, S \neq \emptyset$. We distinguish two cases.

(a) $S \subset S_{0}$. In this case we have $v(S)-\hat{\lambda}(S)<v(S)-\lambda(S) \leq \epsilon_{v}$.

(b) $S=S_{1} \cup S_{2}$, where $S_{1} \subset S_{0}$, and $S_{2} \subset N \backslash S_{0}$ satisfies $S_{2} \neq \emptyset$. Since $S_{2} \neq \emptyset$, we have $\lambda(S)>0$; hence $v(S)-\lambda(S)<\epsilon_{v}$. If $j \notin S$, then $v(S)-\hat{\lambda}(S)$ $\leq v(S)-\lambda(S)<\epsilon_{v}$. If $j \in S$, then $v(S)-\lambda(S) \leq \delta$. Thus

$$
\begin{aligned}
v(S)-\hat{\lambda}(S) & =v(S)-\lambda(S)+\lambda(S)-\hat{\lambda}(S) \\
& \leq \delta+\lambda(S)-\hat{\lambda}(S) \\
& =\lambda\left(S_{1}\right)-\hat{\lambda}\left(S_{1}\right)+\delta+\lambda(\{j\})-\hat{\lambda}(\{j\}) \\
& \leq \delta+\epsilon<\epsilon_{v} .
\end{aligned}
$$

The following example taken from Kannai [9] (Example 2.1) shows that when a game is not continuous its least core may contain only purely finitely additive payoff measures.

Example 3.4. Define a game $v$ by

$$
v(S)= \begin{cases}1 & N \backslash S \text { is finite } \\ 0 & \text { otherwise. }\end{cases}
$$

It is easy to see that the core of $v$ is not empty, and therefore it coincides with the least core. Since a payoff measure $\mu \in b a$ is in the core of $v$ if and only if $\mu(S)=0$ for each finite $S \in 2^{N}$, the least core of $v$ contains only purely finitely additive measures.

Remark. The least core for games with a finite set of players was defined by Maschler, Peleg and Shapley [13] as the set of all payoff measures $\mu$ for which

$$
\min _{\lambda \in I^{*}(v)} \max _{S / \emptyset, S / N}(v(S)-\lambda(S))
$$

is attained at $\lambda=\mu$, where $I^{*}(v)$ is the set of preimputations. It can be easily verified that for games with an empty core our definition applied to a game with a finite set of players coincides with the above definition. However, if $C(v) \neq \emptyset$ then $L C(v)=C(v)$ according to our definition, whereas the original definition may yield a strict subset of the core. In this work we restrict ourselves to solution concepts which are individually rational. Therefore we use our definition which also turn out to be more convenient from the technical point of view.

\section{The kernel}

The set of all payoff measures in a game $v$ is denoted by $I^{*}(v)$; that is

$$
I^{*}(v)=\{\mu \in b a \mid \mu(N)=v(N)\} .
$$


For $i, j \in N, i \neq j$, define the set

$$
\mathscr{T}_{i j}=\left\{S \in 2^{N} \mid i \in S, j \notin S\right\} .
$$

Also for $i, j \in N, i \neq j$, and $\mu \in b a$ define

$$
s_{i j}(\mu)=\sup \left\{v(S)-\mu(S) \mid S \in \mathscr{T}_{i j}\right\} .
$$

The prekernel of $v$ is the set

$$
P K(v)=\left\{\mu \in I^{*}(v) \mid s_{i j}(\mu)=s_{j i}(\mu), \forall i, j \in N, i \neq j\right\} .
$$

The kernel of $v$ is the set

$$
K(v)=\left\{\mu \in I(v) \mid\left(s_{i j}(\mu)-s_{j i}(\mu)\right)\left(\mu(\{j\})-\sigma_{v}(\{j\})\right) \leq 0, \forall i, j \in N, i \neq j\right\} .
$$

The notion of the kernel of a coalitional game with a finite set of players was introduced by Davis and Maschler [5]. It is well-known that if $v$ is a superadditive game with a finite set of players, then $K(v)=P K(v)$ (see Theorem 2.7 in Maschler, Peleg and Shapley [12]). It is also well-known that for such games $K(v) \cap L C(v) \neq \emptyset$. In fact, the nucleolus of $v$ is contained in this intersection (see Corollary 6.7 in Maschler, Peleg and Shapley [13], and Theorem 3 in Schmeidler [16]). Theorem B below establishes that the prekernel (and hence the kernel) and the least core have a non-empty intersection for continuous games.

Theorem B. Let $v$ be a superadditive continuous game. Then $P K(v) \cap$ $L C(v) \neq \emptyset$. In particular, $P K(v)$ contains an individually rational countably additive payoff measure.

We need the following lemmas.

Lemma 4.1. Let $v$ be a superadditive continuous game. Then each function $s_{i j}, i, j \in N, i \neq j$, is norm-continuous on $c a$.

Proof. Let $\mu \in c a$ and let $\left\{\mu_{n}\right\}_{n}^{\infty} \subset c a$ be such that $\lim _{n \rightarrow \infty}\left\|\mu_{n}-\mu\right\|=0$. We show that for each $i, j \in N, i \neq j, \lim _{n \rightarrow \infty} s_{i j}\left(\mu_{n}\right)=s_{i j}(\mu)$. Note that the set $\left\{1_{S} \mid S \in \mathscr{T}_{i j}\right\}$ is a closed (and therefore a compact) subset of $\{0,1\}^{N}$ in the topology of pointwise convergence. Since $v$ is continuous and each $\mu_{n} \in c a, v-\mu_{n}$ attains its maximum on $\mathscr{T}_{i j}$. For each $n \in N$ let $S_{n} \in \mathscr{T}_{i j}$ be such that $s_{i j}\left(\mu_{n}\right)=v\left(S_{n}\right)-\mu_{n}\left(S_{n}\right)$, and similarly let $S \in \mathscr{T}_{i j}$ satisfying $s_{i j}(\mu)=$ $v(S)-\mu(S)$. Since $v(S)-\mu(S) \geq v\left(S_{n}\right)-\mu\left(S_{n}\right)$ for every $n \in N$, we have

$$
\begin{aligned}
v\left(S_{n}\right)-\mu_{n}\left(S_{n}\right) & \leq v\left(S_{n}\right)-\mu_{n}\left(S_{n}\right)+(v(S)-\mu(S))-\left(v\left(S_{n}\right)-\mu\left(S_{n}\right)\right) \\
& =v(S)-\mu(S)+\mu\left(S_{n}\right)-\mu_{n}\left(S_{n}\right) \\
& \leq v(S)-\mu(S)+\left\|\mu-\mu_{n}\right\| .
\end{aligned}
$$

Also we have $v\left(S_{n}\right)-\mu_{n}\left(S_{n}\right) \geq v(S)-\mu_{n}(S)$ for each $n \in N$.

As $\left\|\mu-\mu_{n}\right\| \rightarrow 0, \lim _{n \rightarrow \infty}\left(v\left(S_{n}\right)-\mu_{n}\left(S_{n}\right)\right)=v(S)-\mu(S)$. Hence,

$\lim _{n \rightarrow \infty} s_{i j}\left(\mu_{n}\right)=\lim _{n \rightarrow \infty}\left(v\left(S_{n}\right)-\mu_{n}\left(S_{n}\right)\right)=v(S)-\mu(S)=s_{i j}(\mu)$. 
Lemma 4.2. Let $w$ be a superadditive game with a finite support (i.e., for some $n \geq 1, w(S \cap\{1, \ldots, n\})=w(S)$, for all $\left.S \in 2^{N}\right)$. Then $P K(w) \cap L C(w) \neq \emptyset$.

Proof. W.1.o.g. assume that $w \geq 0$. Let $\Sigma=\left\{S \in 2^{N} \mid S \subset\{1, \ldots, n\}\right\}$, and let $u$ be the restriction of $w$ to $\Sigma$. Since $w$ is superadditive, $u$ is a superadditive game with a finite set of players. Therefore by Theorem 2.7 in Maschler, Peleg and Shapley [12], we have $P K(u)=K(u)$, and by Corollary 6.7 in Maschler, Peleg and Shapley [12], we have $P K(u) \cap L C(u) \neq \emptyset$.

Let $\mu \in P K(u) \cap L C(u)$. Define

$$
\bar{\mu}(\{i\})= \begin{cases}\mu(\{i\}), & 1 \leq i \leq n \\ 0, & \text { otherwise }\end{cases}
$$

Obviously $\bar{\mu} \in \mathrm{ca}$, and therefore there is $S^{*} \in 2^{N}$ such that

$$
w\left(S^{*}\right)-\bar{\mu}\left(S^{*}\right)=\max \left\{w(S)-\bar{\mu}(S) \mid S \in 2^{N}\right\} .
$$

Now,

$$
\begin{aligned}
\epsilon_{w} & \leq w\left(S^{*}\right)-\bar{\mu}\left(S^{*}\right) \\
& =u\left(S^{*} \cap\{1, \ldots, n\}\right)-\mu\left(S^{*} \cap\{1, \ldots, n\}\right) \\
& \leq \epsilon_{u} .
\end{aligned}
$$

Let $\lambda \in L C(w)$; since $\lambda$ minimizes the function $f(\xi)=\sup \{w(S)-\xi(S)$ $\left.\mid S \in 2^{N}\right\}$ on $I(w)$, we have $\lambda(S)=0$ for each $S \subset N \backslash\{1, \ldots, n\}$. Thus

$$
\begin{aligned}
\epsilon_{w} & =\max \left\{w(S)-\lambda(S) \mid S \in 2^{N}\right\} \\
& =\max \{u(Q)-\lambda(Q) \mid Q \in \Sigma\} \\
& \geq \epsilon_{u} .
\end{aligned}
$$

Hence $w\left(S^{*}\right)-\bar{\mu}\left(S^{*}\right)=\epsilon_{w}$, and therefore $\bar{\mu} \in L C(w)$.

Now,

$$
\max \left\{w(S)-\bar{\mu}(S) \mid S \in \mathscr{T}_{i j}\right\}=\max \left\{u(Q)-\mu(Q) \mid Q \in \Sigma \cap \mathscr{T}_{i j}\right\},
$$

and

$$
\max \left\{w(S)-\bar{\mu}(S) \mid S \in \mathscr{T}_{j i}\right\}=\max \left\{u(Q)-\mu(Q) \mid Q \in \Sigma \cap \mathscr{T}_{j i}\right\} .
$$

As $\mu \in P K(u) \cap L C(u)$, we get from (4.1) and (4.2) that $\bar{\mu} \in P K(w) \cap$ $L C(w)$.

With these results at hand we can now complete the proof of Theorem B.

Proof of Theorem B. Assume, w.l.o.g., that $\sigma_{v}$ is identically zero and $v(N)=1$. For every $n \in N$ let $S_{n}=\{1, \ldots, n\}$, and let $v_{n}$ be the game given by $v_{n}(S)=v\left(S \cap S_{n}\right)$. By Lemma 4.2, for each $n \in N$ there is $\mu_{n} \in P K\left(v_{n}\right)$ $\cap L C\left(v_{n}\right)$. As the games $v_{n}$ are continuous, then by Theorem A, $\mu_{n} \in c a$ for each $n$. Let $\bar{M}$ be the weak*-closure in $b a$ of the set $M=\left\{\mu_{n} \mid n \in N\right\}$. We show that $\bar{M}$ is a norm-compact subset of $c a$. This yields that the sequence $\left\{\mu_{n}\right\}_{n 1}^{\infty}$ has a subsequence which converges in the norm to a member of $P K(v) \cap L C(v)$. As $\bar{M}$ is a weak*-compact subset of $b a$, then by Lemma 2.2 it 
suffices to show that $\bar{M} \subset c a$. Moreover, since $M \subset c a$, it suffices to show that each $\mu \in \bar{M} \backslash M$ is a member of $c a$. Let $\mu \in \bar{M} \backslash M$. Then there exists a net $\left\{\mu_{n(\alpha)} \mid \alpha \in D\right\}$ in $M$ which converges to $\mu$. Let $\lambda \in L C(v)$. By Theorem A, $\lambda \in c a$. We distinguish two cases (note that $\lambda \geq 0$ and $\lambda(N)=1$ ).

(1) At least one the following holds:

(1a) The net $\left\{\lambda\left(S_{n(\alpha)}\right) \mid \alpha \in D\right\}$ does not converge to 1 .

(1b) The net $\left\{v\left(S_{n(\alpha)}\right)\right\}$ does not converge to 1 .

(1c) There exists a coalition $S$ such that the net $\left\{v_{n(\alpha)}(S)\right\}$ does not converge to $v(S)$.

We show that if $(1 a)$ is satisfied then $\mu$ is countably additive (the proof that $\mu$ is countably additive when $(1 b)$ or $(1 c)$ are satisfied is similar). Now $(1 a)$ implies that there exists a subnet of $\left\{\lambda\left(S_{n(\alpha)}\right) \mid \alpha \in D\right\}$ that converges to a number $a \in[0,1)$. Assume, w.l.o.g., that $\left\{\lambda\left(S_{n(\alpha)}\right) \mid \alpha \in D\right\}$ itself converges to $a$. Let $\epsilon>0$ be such that $a+\epsilon<1$; then there is $\alpha_{0} \in D$ such that for each $\alpha \succeq \alpha_{0}$ (here $\succeq$ denotes the order relation on $D$ ) we have $\lambda\left(S_{n(\alpha)}\right)<a+\epsilon<1$. As $\lim _{n \rightarrow \infty} \lambda\left(S_{n}\right)=1$, there is $n_{0} \in N$ such that $\lambda\left(S_{n}\right)>a+\epsilon$ for every $n>n_{0}$. Thus, for each $\alpha \succeq \alpha_{0}$ we have $n(\alpha) \leq n_{0}$. Let $S \in 2^{N}$. As $\left\{\mu_{n(\alpha)}\right\}$ converges in the weak ${ }^{*}$-topology to $\mu$, the net $\left\{\mu_{n(\alpha)}(S)\right\}$ converges to $\mu(S)$. Hence there is $\beta_{0} \in D$ such that for each $\beta \succeq \beta_{0}$ we have $\left|\mu_{n(\beta)}(S)-\mu(S)\right|<\epsilon$. From the definition of a net it follows that there is $\gamma \in D$, such that $\gamma \succeq \alpha_{0}$, and $\gamma \succeq \beta_{0}$. Therefore $n(\gamma) \leq n_{0}$, and $\left|\mu_{n(\gamma)}(S)-\mu(S)\right|<\epsilon$. Thus we have shown that if $0<\epsilon<1-a$ is given, then for each $S \in 2^{N}$ there is $1 \leq n \leq n_{0}$ such that $\left|\mu_{n}(S)-\mu(S)\right|<\epsilon$. Since the set $\left\{1, \ldots, n_{0}\right\}$ is finite and $\epsilon$ can be chosen arbitrarily small, there is $1 \leq n \leq n_{0}$ such that $\mu_{n}(S)=\mu(S)$.

We now show that this implies that $\mu \in \mathrm{ca}$. Assume that $\left\{T_{l}\right\}_{l 1}^{\infty} \subset 2^{N}$ is a non-decreasing sequence of coalitions such that $\bigcup_{l}^{\infty} T_{l}=N$. Then for every $l \in N$ there is $1 \leq n \leq n_{0}$ such that $\mu_{n}\left(T_{l}\right)=\mu\left(T_{l}\right)$. Therefore there is a subsequence $\left\{l_{k}\right\}_{k}^{\infty} \subset N$, and $1 \leq n \leq n_{0}$, such that $\mu\left(T_{l_{k}}\right)=\mu_{n}\left(T_{l_{k}}\right)$ for each $k \in N$. As $\mu_{n} \in I\left(v_{n}\right) \cap c a_{+}, \lim _{k \rightarrow \infty} \mu_{n}\left(T_{l_{k}}\right)=1$. Therefore $\lim _{k \rightarrow \infty} \mu\left(T_{l_{k}}\right)=1$. Now $\left\{\mu\left(T_{l}\right)\right\}_{l, ~}^{\infty}$ is a non-decreasing and bounded sequence of real numbers, and therefore it converges. As $\left\{\mu\left(T_{l_{k}}\right)\right\}_{k}^{\infty}$ is a subsequence of $\left\{\mu\left(T_{l}\right)\right\}_{l 1}^{\infty}$ which converges to 1 , we have $\lim _{l \rightarrow \infty} \mu\left(T_{l}\right)=1$. Thus, $\mu \in c a$.

(2) Case (1) does not hold. We show that in this case $\mu \in L C(v)$ and thus by Theorem A it is countably additive. As $\lambda \in L C(v)$, then for each $\alpha \in D$ and all $S \subset N$ we have

$$
v\left(S \cap S_{n(\alpha)}\right)-\lambda\left(S \cap S_{n(\alpha)}\right) \leq \epsilon_{v} .
$$

Since $\lambda\left(S_{n(\alpha)}\right)$ converges to 1 , we assume, w.l.o.g., that $\lambda\left(S_{n(\alpha)}\right)>0$ for each $\alpha \in D$. For any $S \subset N$ and $\alpha \in D$ let

$$
\lambda_{n(\alpha)}(S)=\frac{v\left(S_{n(\alpha)}\right)}{\lambda\left(S_{n(\alpha)}\right)} \lambda\left(S \cap S_{n(\alpha)}\right),
$$

and 


$$
a_{n(\alpha)}=1 \quad \frac{v\left(S_{n(\alpha)}\right)}{\lambda\left(S_{n(\alpha)}\right)} .
$$

Then $\lambda_{n(\alpha)} \in I\left(v_{n(\alpha)}\right)$, and the net $\left\{a_{n(\alpha)}\right\}$ converges to zero. Also for every $S \subset N$ we have

$$
v_{n(\alpha)}(S) \quad \lambda_{n(\alpha)}(S) \leq v\left(S \cap S_{n(\alpha)}\right) \quad \lambda\left(S \cap S_{n(\alpha)}\right)+a_{n(\alpha)} .
$$

Therefore

$$
\epsilon_{v_{n(\alpha)}} \leq \epsilon_{v}+a_{n(\alpha)}
$$

As $\mu_{n(\alpha)} \in L C\left(v_{n(\alpha)}\right)$, for every $S \subset N$ we have

$$
v_{n(\alpha)}(S) \quad \mu_{n(\alpha)}(S) \leq \epsilon_{v}+a_{n(\alpha)} .
$$

Hence for every $S \subset N$ we have

$$
v(S) \quad \mu(S) \leq \epsilon_{v}
$$

Thus $\mu \in L C(v)$. As $v$ is continuous by Theorem A, $L C(v) \subset c a$. Therefore $\mu \in c a$.

We have shown that in any case $\mu \in c a$. Thus, $\bar{M} \subset c a$, and therefore it is norm compact in $\mathrm{ca}$. As the sequence $\left\{\mu_{n} \mid n \in N\right\} \subset \bar{M}$, there is a subse quence $\left\{\mu_{n_{k}}\right\}_{k 1}^{\infty}$ which converges in the norm to a member $\mu$ of $\mathrm{ca}$. As $\lim _{k \rightarrow \infty} \mu\left(S_{n_{k}}\right)=\lim _{k \rightarrow \infty} v\left(S_{n_{k}}\right)=1$, and $\lim _{k \rightarrow \infty} v_{n_{k}}(S)=v(S)$ for each $S \in 2^{N}$, an argument identical to the one given in (2) above yields $\mu \in L C(v)$. Let $i, j \in N, i \neq j$. Since $\mu_{n_{k}} \in P K\left(v_{n_{k}}\right)$ for each $k \in N$, we have $s_{i j}\left(\mu_{n_{k}}\right)=s_{j i}$ $\left(\mu_{n_{k}}\right)$. By Lemma 4.1, $\lim _{k \rightarrow \infty} s_{i j}\left(\mu_{n_{k}}\right)=s_{i j}(\mu)$, and $\lim _{k \rightarrow \infty} s_{j i}\left(\mu_{n_{k}}\right)=s_{j i}(\mu)$. Therefore $s_{i j}(\mu)=s_{j i}(\mu)$, and thus $\mu \in P K(v)$. Hence, $P K(v) \cap L C(v) \neq \emptyset$.

Wesley [21] showed by using non standard analysis that a non negative game $v$ has a non empty kernel (for every coalition structure) if it satisfied the following conditions:

$$
\begin{aligned}
& \text { For each } S \in 2^{N}, \lim _{n \rightarrow \infty}(v(S) \quad v(S \cap\{1, \ldots n\}))=0 \text {; and } \\
& \sum_{i=1}^{\infty} r_{i}<\infty \text {, where } r_{i}=\sup \{v(S \cup\{i\}) \quad v(S) \mid S \subset N \backslash\{i\}\} .
\end{aligned}
$$

We show that (4.3) and (4.4) imply that $v$ is continuous, and then we give an example of a continuous game which does not satisfy (4.4). Thus, Wesley's Theorem is a special case of Theorem B when the coalition structure is $\{N\}$.

Lemma 4.3. Assume that $v$ is a non negative superadditive game which satisfies (4.3) and (4.4). Then $v$ is continuous.

Proof. Let $S \in 2^{N}$. We first show that if $\left\{S_{n}\right\}_{n=1}^{\infty}$ is a non decreasing sequence of coalitions such that $\bigcup_{n}^{\infty} S_{n}=S$, then $\lim _{n \rightarrow \infty} v\left(S_{n}\right)=v(S)$. Indeed, if 
$\left\{S_{n}\right\}_{n 11}^{\infty}$ is such a sequence then for each $n \in N$ there is $\bar{m}(n)$ such that $m \geq \bar{m}(n)$ implies $S \cap\{1, \ldots, n\} \subset S_{m}$. As $v$ is monotonic we have

$$
v(S \cap\{1, \ldots, n\}) \leq v\left(S_{m}\right) \leq v(S),
$$

for every $m \geq \bar{m}(n)$. Thus by (4.3), $\lim _{n \rightarrow \infty} v\left(S_{n}\right)=v(S)$.

Assume now that $\left\{S_{n}\right\}_{n 1}^{\infty}$ is a non-increasing sequence of coalitions such that $\bigcap_{n 1}^{\infty} S_{n}=S$. We show that $\lim _{n \rightarrow \infty} v\left(S_{n}\right)=v(S)$. For $A \subset N$ we define $r(A)=\sum_{i \in A} r_{i}$. Then $r \in c a_{+}$. Let $n \in N$, and let $B \in 2^{N}$ be such that $B \cap S=\emptyset$. We show that $v(S \cup B)-v(B) \leq r(B)$. Define $B_{n}=B \cap\{1, \ldots, n\}$. Then by (4.4)

$$
v\left(S \cup B_{n}\right)-v(S) \leq r\left(B_{n}\right) .
$$

As $B_{n+1} \supset B_{n}$, and $\bigcup_{n}^{\infty} B_{n}=B$, by what we have just shown, $\lim _{n \rightarrow \infty} v\left(S \cup B_{n}\right)=v(S \cup B)$. Since $\lim _{n \rightarrow \infty} r\left(B_{n}\right)=r(B)$, we have

$$
v(S \cup B)-v(S) \leq r(B) .
$$

As $B$ was an arbitrary coalition, for each $n \in N$ we have

$$
0 \leq v\left(S_{n}\right)-v(S)=v\left(S \cup\left(S_{n} \backslash S\right)\right)-v(S) \leq r\left(S_{n} \backslash S\right) .
$$

As $\lim _{n \rightarrow \infty} r\left(S_{n} \backslash S\right)=0$, we have $\lim _{n \rightarrow \infty} v\left(S_{n}\right)=v(S)$.

We give an example of a non-negative continuous game which does not satisfy (4.4).

Example 4.4. For every $0 \leq x \leq 1$ let $f(x)=1-\sqrt{ } 1-x$. Define a measure $\mu \in c a$ by $\mu(S)=c \sum_{i \in S^{\frac{1}{i^{2}}}}$, where $c=\left(\sum_{i}^{\infty} 1^{\frac{1}{i^{2}}}\right){ }^{1}$. For every $S \subset N$ let $v(S)=f(\mu(S))$. Since $f$ is continuous on $[0,1], v$ is continuous. Moreover, as $f$ is convex on $[0,1], v$ is convex; that is,

$$
v\left(S_{1} \cup S_{2}\right)+v\left(S_{1} \cap S_{2}\right) \geq v\left(S_{1}\right)+v\left(S_{2}\right)
$$

for each $S_{1}, S_{2} \subset N$ (see Shapley [18]), and in particular, $v$ is superadditive. For every $i \in N$ we have

$$
r_{i}=\sup \{v(S \cup\{i\})-v(S) \mid S \subset N \backslash\{i\}\} \geq v(N)-v(N \backslash\{i\}) .
$$

As

$$
v(N)-v(N \backslash\{i\})=\frac{\sqrt{ } c}{i},
$$

we have

$$
\sum_{i=1}^{\infty} r_{i} \geq \sqrt{ } c \sum_{i=1}^{\infty} \frac{1}{i}
$$

Thus $\sum_{i 11}^{\infty} r_{i}=\infty$, and (4.4) is not satisfied.

As it was mentioned above, Maschler, Peleg, and Shapley [13] showed that for superadditive games with a finite set of players the kernel coincides with the prekernel. By Theorems B there are always countably additive payoffs measures in the kernel and the prekernel of a continuous superad- 
ditive game on $2^{N}$. Using the compactness of $2^{N}$ the same proofs as those of Theorem 2.4 and Theorem 2.7 in Maschler, Peleg, and Shapley [13] yield.

Proposition 4.5. Let $v$ be a superadditive continuous game. Then every countably additive payoff measure in $P K(v)$ is individually rational.

Proposition 4.6. Let $v$ be a superadditive continuous game. Then

$$
K(v) \cap c a=P K(v) \cap c a .
$$

\section{Bargaining sets}

In this section we establish that the Aumann-Maschler and the Mas-Colell bargaining sets of a continuous game with a countable set of players are nonempty sets which contain the set of all countably additive payoff measures in the prekernel.

Let $v$ be a game, let $\mu \in I(v)$, and let $i, j \in N, i \neq j$, be two players. An objection of $i$ to $j$ in $\mu$ is a pair $(A, \lambda)$ such that $\lambda \in b a, \lambda \geq \sigma_{v}, A \subset N$, $i \in A, j \notin A, \lambda(A) \leq v(A)$, and $\lambda(\{k\})>\mu(\{k\})$ for each $k \in A$. A counterobjection of $j$ to $(A, \lambda)$ is a pair $(B, \xi)$ such that $\xi \in b a, \xi \geq \sigma_{v}, B \subset N, j \in B$, $i \notin B, \xi(B) \leq v(B), \xi(\{k\}) \geq \mu(\{k\})$ for $k \in B \backslash A$, and $\xi(\{k\}) \geq \lambda(\{k\})$ for $k \in A \cap B$. A justified objection of $i \in N$ to $j$ in $\mu$ is an objection which does not have a counterobjection. The Aumann-Maschler bargaining set (see Aumann and Maschler [1]) of $v$ is the set $B(v)$ of all imputations $\mu \in I(v)$ such that no player $i$ has a justified objection to another player in $\mu$.

Davis and Maschler [4], and Peleg [15] showed that if $v$ is a game with a finite set of players, then $B(v)$ is a non-empty set. For this class of games, Davis and Maschler [5] proved that $K(v)$ is a subset of $B(v)$. Theorem $\mathrm{C}$ below establishes that if $v$ is a continuous game, then the set of all countably additive payoff measures in the prekernel is contained $B(v)$.

Theorem C. Let $v$ be a superadditive continuous game. Then $P K(v) \cap c a$ $\subset B(v)$. In particular, $B(v)$ contains a countably additive payoff measure.

Proof. W.l.o.g. assume that $\sigma_{v}$ is identically zero. Let $\mu \in P K(v) \cap c a$. We show that $\mu \in B(v)$. Assume to the contrary that $\mu \notin B(v)$. Then there are $i, j \in N, i \neq j$, such that $j$ has a justified objection $(A, \lambda)$ to $i$ in $\mu$. By the Yosida and Hewitt Theorem [22], $\lambda$ can be uniquely decomposed into a sum of a non-negative countably additive measure $\lambda_{c}$, and a non-negative purely finitely additive measure $\lambda_{p}$ (note that $\lambda \geq \sigma_{v}$, and thus $\lambda \geq 0$ ). As $\lambda_{p}$ vanishes on finite subsets of $N$, we have $\lambda_{c}(\{k\})>\mu(\{k\})$ for each $k \in A$, and $\lambda_{c}(A) \leq \lambda(A) \leq v(A)$. Since any counterobjection to $\left(A, \lambda_{c}\right)$ is also a counterobjection to $(A, \lambda),\left(A, \lambda_{c}\right)$ is a justified objection. Since $v$ is continuous and $\mu \in c a$, there is $B \in 2^{N}$ such that $i \in B, j \notin B$, and

$$
s_{i j}(\mu)=v(B)-\mu(B) .
$$


As $\mu \in P K(v)$,

$$
v(B)-\mu(B)=s_{j i}(\mu) \geq v(A)-\mu(A) .
$$

Since $\lambda_{c}(\{j\})>\mu(\{j\})$,

$$
\begin{aligned}
v(A)-\mu(A) & \geq \sum_{k \in A}\left(\lambda_{c}(\{k\})-\mu(\{k\})\right) \\
& >\sum_{k \in A \cap B}\left(\lambda_{c}(\{k\})-\mu(\{k\})\right) .
\end{aligned}
$$

Therefore

$$
v(B)-\sum_{k \in A} \mu(\{k\})>\sum_{k \in A \cap B}\left(\lambda_{c}(\{k\})-\mu(\{k\})\right) ;
$$

i.e.,

$$
v(B)>\sum_{k \in A \cap B} \lambda_{c}(\{k\})+\sum_{k \in B \backslash A} \mu(\{k\}) .
$$

For each $S \subset N$ let $\xi(S)=\lambda_{c}(S \cap A)+\mu(S \cap(B \backslash A))$. Then $\xi(B) \leq v(B)$ by the last inequality, and if $k \in A \cap B, \xi(\{k\})=\lambda_{c}(\{k\})$, and if $l \in B \backslash A$, then $\xi(\{l\})=\mu(\{l\})$. Therefore each member of $B$ (and in particular player $i$ ) has a counterobjection to the objection $\left(A, \lambda_{c}\right)$ of $j$, which contradicts the fact that $\left(A, \lambda_{c}\right)$ is a justified objection. Now by Theorem A, $P K(v) \cap L C(v) \subset$ $P K(v) \cap c a$. Therefore by Theorem B, $B(v)$ contains a countably additive payoff measure.

Mas Colell [14] proposed a notion of bargaining set different from that of Aumann and Maschler bargaining set, and showed that in the context of a market with a continuum of players, this new bargaining set coincides with the set of walrasian allocations. The advantage of the Mas-Colell bargaining set is that it can be defined for games with an uncountable set of players.

The following definitions are taken from Einy et al. [8], who provide a straightforward generalization of the Mas-Colell [14] definition to games with an infinite set of players. Let $v$ be a game, and let $\mu$ be a payoff measure in $v$. An objection to $\mu$ (in the sense of Mas-Colell) is a pair $(A, \lambda)$ such that $A \in 2^{N}$, and $\lambda \in b a$ satisfies $\lambda(A) \leq v(A), \lambda(A)>\mu(A)$, and $\lambda(B) \geq \mu(B)$ for every coalition $B \subset A$. A counterobjection (in the sense of Mas-Colell) to the objection $(A, \lambda)$ is a pair $(C, \xi)$ such that

$$
\begin{aligned}
& \xi \in b a, \text { and } \xi(C) \leq v(C) \\
& \text { For every } B \subset A \cap C, \xi(B) \geq \lambda(B), \text { and for every } D \subset C \backslash A, \\
& \qquad \xi(D) \geq \mu(D) ; \text { and } \\
& \xi(C)>\lambda(A \cap C)+\mu(C \backslash A)
\end{aligned}
$$

A justified objection is an objection which has no counterobjection. The MasColell bargaining set of $v$ is the set $M B(v)$ of all payoff measures which have no justified objection. 
Mas-Colell [14] in considering exchange economies with a continuum of agents, defines the bargaining set without restricting attention to individually rational allocations. Thus, his equivalence result holds for a large set. From the point of view of an existence result it will be interesting to show that the Mas-Colell bargaining set always contains an individually rational payoff measure. Theorem D below establishes that the Mas-Colell bargaining set of a continuous game contains the set of all countably additive payoff measures in the prekernel. As a consequence, for these games the Mas-Colell bargaining set contains an individually rational countably additive payoff measure.

Theorem D. Let $v$ be a superadditive continuous game. Then $P K(v) \cap c a$ $\subset M B(v)$. In particular, $M B(v)$ contains an individually rational countably additive payoff measure.

The following lemma will be useful in the proof of Theorem D.

Lemma 5.1. Let $v$ be game, and let $\mu$ be a payoff measure in $v$. If $(A, \lambda)$ is a justified objection to $\mu$, then $v(B) \leq \lambda(B \cap A)+\mu(B \backslash A)$ for each $B \in \Sigma$.

Proof. Assume to the contrary that there is $B \in \Sigma$ such that $v(B)>\lambda(B \cap A)$ $+\mu(B \backslash A)$. Then $B \neq \emptyset$. Let $\epsilon=v(B)-(\lambda(B \cap A)+\mu(B \backslash A))$. Choose $t \in B$, and let $\delta_{t}$ be the probability measure concentrated on $\{t\}$. Define $\xi \in b a$ by

$$
\xi(S)=\lambda(S \cap(B \cap A))+\mu(S \cap(B \backslash A))+\epsilon \delta_{t}(S) .
$$

Thus, if $S \in \Sigma$ satisfies $S \subset A \cap B$ we have $\xi(S) \geq \lambda(S)$, and if $S \in \Sigma$ satisfies $S \subset B \backslash A$ we have $\xi(S) \geq \mu(S)$. Also

$$
\xi(B)=v(B)>\lambda(B \cap A)+\mu(B \backslash A) .
$$

Thus $(B, \xi)$ is a counterobjection to $(A, \lambda)$, which is a contradiction.

Proof of Theorem D. W.l.o.g. assume that $\sigma_{v}$ is identically zero. Let $\mu \in P K(v) \cap c a$. We show that $\mu \in M B(v)$. Assume by way of contradiction that $\mu \notin M B(v)$. Then there is a justified objection $(A, \lambda)$ to $\mu$. Since $\mu$ is a payoff measure, $A \neq N$. Assume, w.l.o.g., that $\lambda(A)=v(A)$. Since $(A, \lambda)$ is a justified objection to $\mu$, by Lemma 5.1 for each $B \subset A$ we have $\lambda(B) \geq v(B)$. For each $S \in 2^{N}$ let $v_{A}(S)=v(S \cap A)$, and $\lambda_{A}(S)=\lambda(S \cap A)$. Then $\lambda_{A}$ is in the core of the game $v_{A}$. Since $v_{A}$ is continuous, $\lambda_{A} \in c a$. As $\lambda(A)>\mu(A)$, there is $j \in A$ such that $\lambda_{A}(\{j\})>\mu(\{j\})$. Let $i \in N \backslash A$. As $\mu \in P K(v)$, there is $C \subset N, i \in C, j \notin C$, such that $v(C)-\mu(C)=s_{i j}(\mu) \geq v(A)-\mu(A)$. Since $\lambda_{A}(\{j\})>\mu(\{j\})$, an argument identical to the one given in the proof of Theorem $\mathrm{C}$ yields

$$
\begin{aligned}
v(C) & >\sum_{k \in A \cap C} \lambda_{A}(\{k\})+\sum_{k \in C \backslash A} \mu(\{k\}) \\
& =\lambda(A \cap C)+\mu(C \backslash A) .
\end{aligned}
$$


By Lemma 5.1 this contradicts the assumption that $(A, \lambda)$ is a justified objection to $\mu$. Now by Theorem $\mathrm{A}, P K(v) \cap L C(v) \subset M B(v)$, and thus by Theorem $\mathrm{B}, M B(v)$ contains an individually rational countably additive payoff measure.

\section{References}

1. Aumann, R.J., Maschler, M.: The bargaining set for cooperative games. In: Dresher, M., Shapley, L.S., Tucker, A.W. (eds.) Advances in Game Theory, pp. 443-476. Princeton, N.J.: Princeton University Press 1964

2. Aumann, R.J., Shapley, L.S.: Values of nonatomic games. Princeton, N.J.: Princeton University Press 1974

3. Bird, C.G.: Extending the nucleolus to infinite player games. SIAM Journal of Applied Mathematics 31, 474- 484 (1976)

4. Davis, M., Maschler, M.: Existence of stable payoff configuration for cooperative games. Bulletin of the American Mathematical Society 69, 106-108 (1963)

5. Davis, M., Maschler, M.: The kernel of a cooperative games. Naval Research Logistic Quarterly 12, 223-259 (1965)

6. Diestel, J.: Sequences and series in Banach spaces. New York: Springer 1984

7. Dunford, N., Schwartz, J.T.: Linear operators, Part I: General Theory. New York: John Wiley 1988

8. Einy, E., Holzman, R., Monderer, D., Shitovitz, B.: Core equivalence theorems for infinite convex games. Journal of Economic Theory (forthcoming) (1996)

9. Kannai, M.: The core and balancedness. In: R.J. Aumann, S. Hart (eds.) Handbook of Game Theory, vol. 1, pp. 543-590. Amsterdam: Elsevier 1992

10. Kelley, J.L.: General Topology. Toronto: van Nostrand 1969

11. Maschler, M.: The bargaining set, kernel, and nucleolus. In: R.J. Aumann, S. Hart (eds.) Handbook of Game Theory, vol. 1, pp. 591-667. Amsterdam: Elsevier 1992

12. Maschler, M., Peleg, B., Shapley, L.S.: The kernel, and the bargaining set for convex games. International Journal of Game Theory 1, 73-93 (1972)

13. Maschler, M., Peleg, B., Shapley, L.S.: Geometric properties of the kernel, nucleolus and related solution concepts. Mathematics of Operations Research 4, 303-338 (1979)

14. Mas-Colell, A.: An equivalence theorem for a bargaining set. Journal of Mathematical Economics 18, 124-138 (1989)

15. Peleg, B.: Existence theorem for the bargaining set $M_{1}^{(i)}$. Bulletin of the American Mathematical Society 69, 109-110 (1963)

16. Schmeidler, D.: The nucleolus of a characteristic function game. SIAM Journal of Applied Mathematics 17, 1163-1170 (1969)

17. Schmeidler, D.: Cores of exact games, I. Journal of Mathematical Analysis and Applications 40, 214-225 (1972)

18. Shapley, L.S.: Cores of convex games. International Journal of Game Theory 1, 11-26 (1971)

19. Shitovitz, B.: The bargaining set and the core in mixed markets with atoms and atomless sector. Journal of Mathematical Economics 18, 377-383 (1989)

20. Vorha, R.: An existence theorem for a bargaining set. Journal of Mathematical Economics 20, 19-34 (1991)

21. Wesley, E.: An application of non-standard analysis to game theory. Journal of Symbolic Logic 36, 385-394 (1971)

22. Yosida, K., and Hewitt, E.: Finitely additive measures. Transactions of American Mathematical Society 72, 46-66 (1952) 